\title{
Herzgesunde Städte - die Gene laden das Gewehr, die Umwelt zieht den Abzug
}

\section{Heart Healthy Cities - Genes Load the Gun, the Environment Pulls the Trigger}

\section{(ㄷ) (2) (1) (5)}

\author{
Autoren

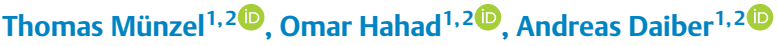 \\ Institute \\ 1 Zentrum für Kardiologie, Kardiologie I, Universitätsmedizin \\ der Johannes Gutenberg-Universität Mainz, Mainz, \\ Deutschland \\ 2 Deutsches Zentrum für Herz-Kreislauf-Forschung (DZHK), \\ Standort Rhein-Main, Mainz, Deutschland
}

\section{Schlüsselwörter}

Umweltrisikofaktoren, Stadtplanung, herzgesunde Städte

Key words

environmental risk factors, urban planning, heart healthy cities

\section{Bibliografie}

Aktuel Kardiol 2021; 10: 543-547

DOI 10.1055/a-1546-7383

ISSN 2193-5203

(c) 2021. The Author(s).

This is an open access article published by Thieme under the terms of the Creative Commons Attribution-NonDerivative-NonCommercial-License, permitting copying and reproduction so long as the original work is given appropriate credit. Contents may not be used for commercial purposes, or adapted, remixed, transformed or built upon. (https://creativecommons.org/licenses/by-nc-nd/4.0/).

Georg Thieme Verlag KG, Rüdigerstraße 14,

70469 Stuttgart, Germany

Korrespondenzadresse

Prof. Thomas Münzel

Zentrum für Kardiologie, Kardiologie I

Universitätsmedizin der Johannes Gutenberg-Universität

Mainz

Langenbeckstraße 1

55131 Mainz, Deutschland

tmuenzel@uni-mainz.de

\section{ZUSAMMENFASSUNG}

Die Weltgesundheitsorganisation gibt an, dass die Stadtplanung mittlerweile als kritischer Bestandteil einer umfassenden Lösung zur Bekämpfung umweltbedingter Gesundheitsschäden gesehen wird. Die vorliegende Übersicht befasst sich mit nicht übertragbaren Krankheiten mit dem Schwerpunkt auf Herz-Kreislauf-Erkrankungen und dem Urbanisierungsprozess in Bezug auf Umweltrisiken wie Lärm, Luftverschmutzung, Temperatur und Licht im Freien. Es wird dargelegt, warum sich Wärmeinseln in städtischen Gebieten entwickeln können und wie die Begrünung von Städten die öffentliche Gesundheit verbessern und Klimaprobleme, Nachhaltigkeit und Lebensfähigkeit adressieren kann. Darüber hinaus thematisieren wir Stadtplanung, Verkehrsinterventionen und neuartige Technologien zur Bewertung externer Umweltexpositionen, z. B. Einsatz digitaler Technologien, um zukünftig herzgesunde Städte zu fördern. Zuletzt heben wir neue Paradigmen des integrativen Denkens hervor, wie das Exposom und die planetare Gesundheit, indem wir den Zusammenhang zwischen Exposition und Gesundheitsauswirkungen kritisch evaluieren und unser Verständnis der Gesamtheit der Expositionen gegenüber der Umwelt des Menschen erweitern.

\section{ABSTRACT}

The World Health Organization states that urban planning is now recognized as a critical part of a comprehensive solution to address harmful health effects of the environment. The present review deals with non-communicable diseases with an emphasis on cardiovascular diseases and the urbanization process in relation to environmental risks such as noise, air pollution, temperature and outdoor light. It discusses why heat islands can develop in urban areas and how green spaces in cities can improve public health and address climate issues, sustainability and viability. In addition, we discuss urban planning, traffic interventions and novel technologies for assessing external environmental exposures, e.g. use of digital technologies to promote heart-healthy cities in the future. Lastly, we highlight new paradigms of integrative thinking, such as the exposome and planetary health, by critically evaluating the relationship between exposure and health outcomes and by broadening our understanding of the totality of exposures to the human environment. 


\section{WAS IST WICHTIG?}

Bis 2050 werden ca. 7,5 Milliarden Menschen in Städten leben und für einen Großteil der Umweltbelastungen verantwortlich sein, die in Verbindung mit erhöhten vorzeitigen Todesfällen durch nicht übertragbare Krankheiten wie koronare Herzerkrankungen, Schlaganfälle, Diabetes mellitus und Bluthochdruck stehen. Durch nachhaltige Konzepte der Stadtplanung, die auf mehr begrünte Flächen, weniger Hitzeinseln und mehr Kompaktheit in Kombination mit autofreien Zonen und vermehrtem Gebrauch von öffentlichen Verkehrsmitteln und Fahrrädern abzielen, können herzgesunde Umgebungen geschaffen werden.

\section{Einleitung}

Die Weltbevölkerung wird bis 2050 auf 10 Milliarden Menschen wachsen, von denen $75 \%$ in Städten leben werden. 60-80\% des weltweiten Endenergieverbrauchs findet in städtischen Gebieten statt und $70 \%$ der Treibhausgasemissionen werden in Städten erzeugt. Mehr als $90 \%$ der Weltbevölkerung atmen mit Feinstaub (PM2,5) belastete Außenluft, die über dem Grenzwert der Weltgesundheitsorganisation $(\mathrm{WHO})$ liegt $\left(10 \mu \mathrm{g} / \mathrm{m}^{3}\right)$. Die Folge ist, dass die Umweltverschmutzung im Zuge der erweiterten Urbanisierung erheblich zunehmen wird. Die WHO hat daher postuliert, dass aufgrund der hohen Anzahl von Menschen, die in Städten leben, die städtische Umgebung sowie ein gesunder Lebensstil wichtige Voraussetzungen für ein gesundes Herz sind [1, 2].

\section{Durch Städteplanung beeinflussbare Gesundheitsrisiken}

Insgesamt wurden mehrere städtische Gesundheitsrisiken identifiziert, die das Entstehen von Herz-Kreislauf-Erkrankungen begünstigen. Dazu gehören der Verkehr in der Stadt, die Luftverschmutzung und der Lärm, die soziale Isolation, die persönliche Sicherheit, ungesunde Ernährung, längeres Sitzen sowie körperliche Inaktivität. In dieser Übersicht sollen primär die Umweltgesundheitsrisiken wie Luftverschmutzung und Lärm sowie die gesundheitlichen Auswirkungen von sogenannten Wärmeinseln und Grünflächen auf die Entstehung von Herz-Kreislauf-Erkrankungen diskutiert werden [2, 3].

\section{Gesundheitliche Belastung durch Luftverschmutzung}

Unter Luftverschmutzung versteht man eine komplexe Mischung einer Vielzahl von gasförmigen, flüssigen und festen Komponenten. Hier soll vor allem der Feinstaub erwähnt werden, der als besonders gesundheitsschädlich gilt. Entscheidend dabei ist, dass, je kleiner der Feinstaubpartikel ist, desto größer ist seine Fähigkeit, in die Zirkulation zu gelangen und von verschiedenen Organen aufgenommen zu werden, um dort gesundheitliche Schäden auszulösen [4]. Laut unseren Schätzungen liegt die europäische Übersterblichkeit aufgrund der Feinstaubbelastung bei jährlich etwa 600000 Menschen, wobei die globale Übersterblichkeit aufgrund von Luftverschmutzung jährlich etwa bei 8,79 Millionen liegt, vor allem bedingt durch Herz-Kreislauf-Erkrankungen wie koronare Herzerkrankungen, Schlaganfall und andere nicht übertragbare Erkrankungen wie Diabetes mellitus und Bluthochdruck [5]. In der Stadt ist der Kraftfahrzeugverkehr die wichtigste Emissionsquelle. Menschen, die in einem näheren Umkreis von stark befahrenen Straßen leben, sind höheren Schadstoffgehalten, einschließlich Feinstaub, Kohlenmonoxid und Stickstoffdioxid, ausgesetzt. In Ländern mit geringeren Einkommen sind die Autos typischerweise älter und verursachen daher höhere Emissionen als Autos in Ländern mit höheren Durchschnittseinkommen [3].

\section{KURZGEFASST}

Luftverschmutzung ist ein globales Gesundheitsproblem, die für eine globale Übersterblichkeit von jährlich etwa 8,79 Millionen Menschen verantwortlich ist, vor allem bedingt durch Herz-Kreislauf-Erkrankungen.

\section{Maßnahmen zur Vermeidung von \\ Gesundheitsrisiken durch Luftverschmutzung}

Körperliche Aktivität im Freien kann die Exposition gegenüber Luftverschmutzung (abhängig von den ortsspezifischen aktuellen Luftqualität) unter Umständen weiter erhöhen und so die allgemeinen gesundheitlichen Vorteile der körperlichen Aktivität sogar umkehren. Eine innovative Stadtplanung kann hier hilfreich sein, indem Häuser, Schulen, Parks und Übungsanlagen abseits stark befahrener Straßen gebaut werden und indem man strikt Radwege vom Kraftfahrzeugverkehr trennt [6]. Auch digitale Technologien wie Smartphones und -watches, die aktuelle Feinstaubkonzentrationen messen und individuell signalisieren, ob körperliche Aktivität im Freien aus gesundheitstechnischen Aspekten sinnvoll ist, können die gesundheitlichen Vorteile durch körperliche Aktivität im städtischen Raum unterstützen ( Abb. 1) [6, 7]. Weitere persönliche Maßnahmen zur Vermeidung von Gesundheitsrisiken durch Luftverschmutzung sind in > Abb. 2 zusammengefasst.

\section{KURZGEFASST}

Maßnahmen zur Reduktion der gesundheitlichen Folgen von Luftverschmutzung sind zwingend notwendig. Um die Exposition gegenüber Luftverschmutzung zu verringern, können Stadtplanungsmaßnahmen hilfreich sein, die dafür sorgen, dass z. B. Häuser, Schulen und Parks abseits stark befahrener Straßen gebaut werden.

\section{Gesundheitliche Belastung durch Lärm}

Umgebungslärm, der hauptsächlich in städtischen Gebieten mit Luftverschmutzung einhergeht, wurde bisher wesentlich weniger beachtet als Luftverschmutzung, obwohl täglich fast 70 Millionen Europäer in Städten und Gemeinden Verkehrslärmpegeln von mehr als 55 Dezibel ausgesetzt sind und damit einem Pegel, ab 


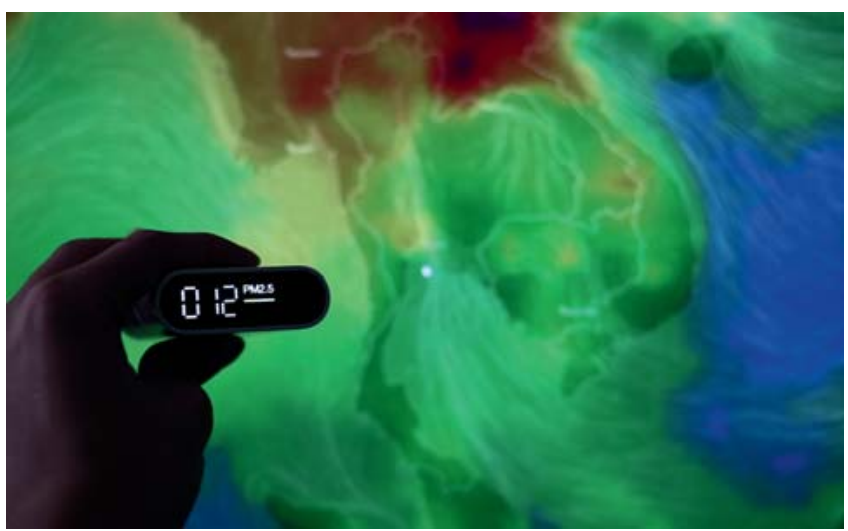

- Abb. 1 Feinstaubbelastung messen (Quelle: (c) suebsiri/ stock.adobe.com).

dem man mit einer erhöhten Inzidenz von Herz-Kreislauf-Erkrankungen rechnen muss. Für die Europäische Union wird geschätzt, dass Verkehrslärm zusätzlich zu 900000 Fällen von Bluthochdruck, 43000 Krankenhauseinweisungen und mehr als 10000 vorzeitigen Todesfällen pro Jahr im Zusammenhang mit koronaren Herzerkrankungen und Schlaganfällen führt [7]. Straßenverkehrslärm ist hierbei die wichtigste Quelle für Umgebungslärm weltweit. Entsprechend dem Lärmwirkungsmodell von Babisch führen insbesondere Lärmpegel im Bereich von 50-60 Dezibel über die indirekte Lärmwirkung zu physiologischen und mentalen Stressreaktionen, vermittelt über lärmbedingte Beeinträchtigungen von Kommunikation, Aktivitäten und des Schlafes, die in Lärmbelästigung resultieren. So wurde gezeigt, dass Lärmbelästigung mit einem erhöhten Risiko für Herzrhythmusstörungen (Vorhofflimmern), aber auch für psychische Erkrankungen wie Depressionen, Angststörungen sowie kognitiven Störungen vor allem bei Kindern einhergeht (für eine Übersicht siehe [8]).

\section{KURZGEFASST}

Umgebungslärm betrifft einen Großteil der städtischen Bevölkerung und ist ein manifester Herz-Kreislauf-Risikofaktor, der chronische physiologische und mentale Stressreaktionen hervorruft.

\section{Maßnahmen zur Lärmreduktion in der Stadt}

Um die gesundheitlichen Auswirkungen der Lärmbelastung zu reduzieren, muss eine bessere Isolierung von Häusern und Schulen angestrebt werden. Eine Reduktion des Transportverkehrs sowie ein generelles Tempolimit, geräuschmindernde Straßenbeläge, Flüsterbremsen bei Zügen, Lärmschutzwände sowie ein verbessertes Verkehrsmanagement können als effektive Lärmschutzmaßnahmen dienen. > Tab. 1 zeigt einen Überblick über die Lärmreduktions- und Kosteneffektivität in Abhängigkeit von spezifischen Lärmschutzmaßnahmen [8].

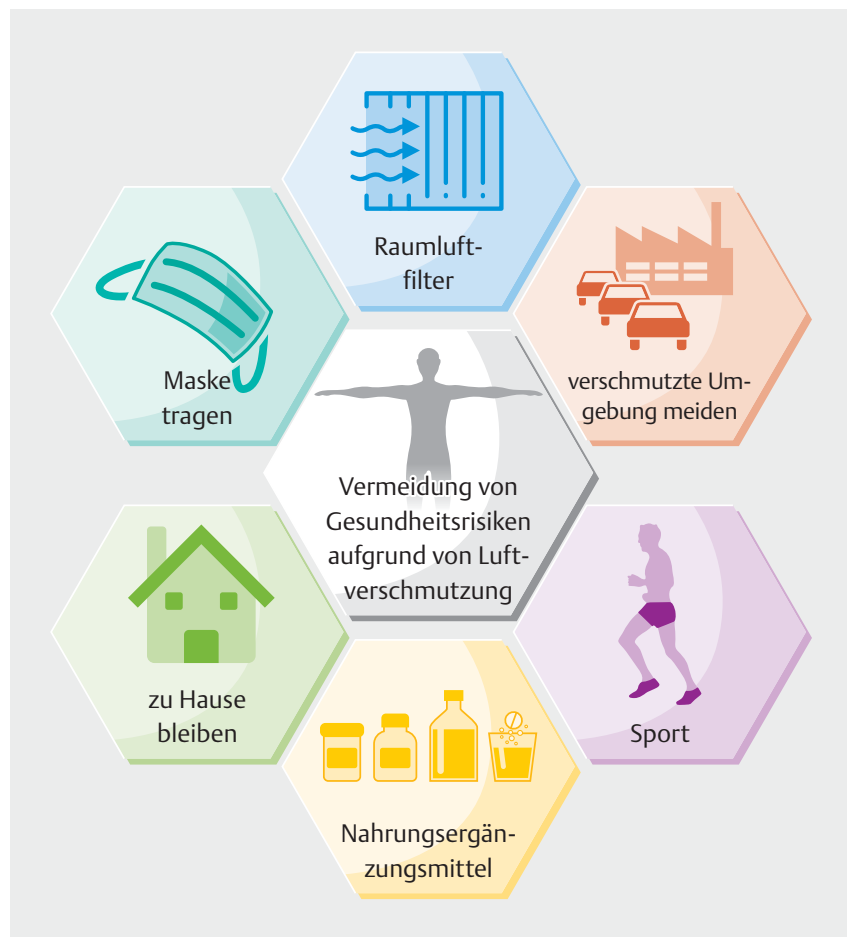

- Abb. 2 Individuelle Maßnahmen, um die gesundheitlichen Folgen von Luftverschmutzung zu mildern [7].

Tab. 1 Lärmschutzmaßnahmen (evaluiert durch die Europäische Kommission). Niedrigste (1) bis höchste (5) Kosteneffektivität. dB: Dezibel. Daten aus [8].

\begin{tabular}{|l|l|l|}
\hline Lärmschutzmaßnahme & $\begin{array}{l}\text { Reduktion des } \\
\text { Lärms (in dB) }\end{array}$ & $\begin{array}{l}\text { Kosteneffek- } \\
\text { tivität (1-5) }\end{array}$ \\
\hline Lärmschutzwände & $3-20$ & 2 \\
\hline Flüsterbremsen für Züge & $8-10$ & 4 \\
\hline Hausisolierung & $5-10$ & 1 \\
\hline Hausdesign & $2-15$ & 3 \\
\hline veränderte Fahrweise & $5-7$ & 3 \\
\hline lärmreduzierende Straßenbeläge & $3-7$ & 5 \\
\hline lärmreduzierende Reifen & $3-4$ & 3 \\
\hline Landnutzungsplanung und & unbekannt & 4 \\
\hline -gestaltung & & 1 \\
\hline elektrische Autos & 1 & 3 \\
\hline Verkehrsmanagement & 3 & \\
\hline
\end{tabular}

\section{KURZGEFASST}

Städtische Lärmschutzmaßnahmen sind notwendig, um die Bevölkerung vor den negativen gesundheitlichen Konsequenzen zu schützen. 


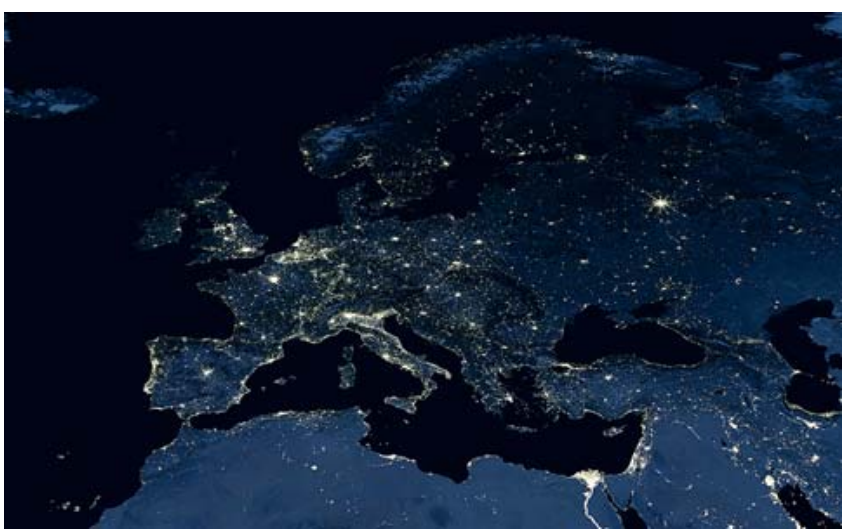

- Abb. 3 Lichtverschmutzung (Quelle: @ scaliger/stock.adobe.com).

\section{Nächtliches Licht im Freien}

Schätzungen zufolge sind über $80 \%$ der Weltbevölkerung in der Nacht einem „lichtverschmutzten“ Himmel ausgesetzt ( [9], was mit einem höheren Krebsrisiko, Übergewicht, erhöhtem Blutdruck, Depressionen, Schlaflosigkeit und dem vermehrten Auftreten einer koronaren Herzerkrankung in Verbindung gebracht wird. In einer aktuellen Studie wurde gezeigt, dass bei älteren Erwachsenen nächtliches Licht im Freien mit einem erhöhten Risiko für die Entwicklung einer koronaren Herzerkrankung, vermehrten Krankenhauseinweisungen und Todesfällen verknüpft ist [10]. Die gesundheitsschädlichen Auswirkungen der nächtlichen Lichtverschmutzung werden vermutlich über ähnliche Pathomechanismen vermittelt, wie sie für die nächtliche Verkehrslärmexposition nachgewiesen wurden, d.h. Stressreaktionen durch Schlaffragmentierung und -entzug verbunden mit einer Störung des zirkadianen Rhythmus [2].

KURZGEFASST

Nächtliches Licht im Freien verursacht über ähnliche Pathomechanismen wie nächtlicher Verkehrslärm ein erhöhtes Risiko für Herz-Kreislauf-Erkrankungen.

\section{Gesundheitliche Auswirkungen von Wärmeinseln und Grünflächen}

Der städtische Wärmeinseleffekt wird häufig dort beobachtet, wo offene, bewaldete oder grüne Flächen durch Beton und Asphalt ersetzt wurden. Bevölkerungsdichte, Vegetation, Städtebau und Rückstrahlungseffekte können zu Temperaturunterschieden führen. Diese können zwischen städtischen und angrenzenden ländlichen Gebieten bis zu $3-5{ }^{\circ} \mathrm{C}$ betragen. Die innerstädtische Variabilität im Sommer beträgt sowohl im Minimum als auch im Maximum fast $3{ }^{\circ} \mathrm{C}$. Hohe und niedrige Umgebungstemperaturen wurden mit einer erhöhten Letalität, kardiorespiratorischen Morbidität und mehr Krankenhauseinweisungen in Verbindung gebracht [3]. Grünflächen sind mit einer Reihe von positiven gesundheitlichen Auswirkungen verbunden wie reduzierte Letalität und erhöhte Le- benserwartung, weniger Herz-Kreislauf-Erkrankungen, psychische Gesundheit, weniger Schlafstörungen, mehr soziale Kontakte und positive Auswirkungen auf Mikroorganismen und Geburtsraten. Erhöhte körperliche Aktivität und verbesserte soziale Kontakte, Stressabbau und eine Reduzierung von schädlichen Umwelteinflüssen wie Lärm und Luftverschmutzung und Wärme werden hierbei als Mechanismen für die gesundheitlichen Vorteile von Grünflächen angeführt $[2,6]$.

\section{Neue Städtemodelle}

In verschiedenen Städten wird eine Reihe neuer urbaner Konzepte eingeführt, welche die oben beschriebenen Probleme bis zu einem gewissen Grad adressieren, z. B. die kompakte Stadt, Superblöcke, 15-Minuten-Stadt, autofreie Stadt oder eine Mischung aus diesen. Kompakte Städte sind Städte mit höherer Dichte, kürzeren Wegen und höherer Vielfalt. Im Vergleich zu zersiedelten Städten mit geringer Dichte sind ihre $\mathrm{CO}_{2}$-Emissionen geringer, weil sie einen besseren Flächennutzungsmix und kürzere und nachhaltige Mobilitätsmöglichkeiten haben. Wenn Städte um 30\% kompakter werden, könnten je nach Stadttyp jährlich etwa 400-800 verlorene gesunde Lebensjahre (DALYs) pro 100000 Menschen durch Verhinderung von nicht übertragbaren Krankheiten vermieden werden.

In Barcelona sind über 500 Superblöcke geplant, die den motorisierten Verkehr in einigen Straßen eines Blockes reduzieren und Platz für Menschen, aktive Fortbewegung und Grünflächen bieten. Dies reduziert die Luftverschmutzung, den Lärmpegel sowie den Hitzeinseleffekt, vergrößert die Grünflächen und erhöht die körperliche Aktivität, wodurch fast 700 vorzeitige Todesfälle pro Jahr in Barcelona verhindert werden [11].

Paris führt die 15-Minuten-Stadt ein, in der Arbeit, Schule, Unterhaltung und andere Aktivitäten innerhalb eines 15-minütigen Fußwegs von der Wohnung aus erreichbar sind [2]. So werden Langstreckenfahrten und damit der $\mathrm{CO}_{2}$-Ausstoß, die Luftverschmutzung und die Lärmbelastung drastisch reduziert. Die 15Minuten-Stadt beinhaltet die Schaffung einer "Stadt der Dörfer“ und eine Rückkehr zu einem traditionelleren Stadtdesign [12]. Die 15-Minuten-Stadt wird zu mehr körperlicher Aktivität ermutigen und wahrscheinlich städtische Ungleichheiten und gesundheitliche Ungleichheiten reduzieren.

Hamburg plant, bis 2034 eine autofreie Stadt zu werden, auch um die Klimakrise zu bekämpfen. Autofreie Städte reduzieren unnötigen motorisierten Individualverkehr und bieten einen einfachen Zugang zu aktiven und öffentlichen Verkehrsmitteln. Sie reduzieren die Luftverschmutzung und die Lärmbelastung, erhöhen die körperliche Aktivität und schaffen Raum für Grünflächen. Ein erfolgreiches Beispiel ist Vauban in Freiburg, Deutschland, ein Viertel ohne Autos und mit nachhaltigem Wohnraum.

\section{Zusammenfassung und politische Konsequenzen}

Derzeit fließen etwa 96\% der öffentlichen Gesundheitskosten in die Behandlung und nur $4 \%$ in die Prävention. Darüber hinaus sind die für die Prävention bereitgestellten Gelder überwiegend auf die individuelle Ebene gerichtet, wobei nur sehr wenig Aufwand und Geld für soziale und landschaftliche Maßnahmen zur Minderung 
von Umweltbelastungen aufgewendet werden. In diesem Sinne sind Investitionen zur Erleichterung breit angelegter Maßnahmen zur Verbesserung unserer Städte dringend erforderlich, um die Gesundheit der Bevölkerung zu schützen. Die erfolgreiche Umsetzung dieser Bemühungen, das Risiko für Herz-Kreislauf-Erkrankungen und Krebs zu reduzieren und eine gesunde und nachhaltige Zukunft zu schaffen, erfordert ein radikales Umdenken darüber, wie wir die zukünftige Art und Weise unseres Zusammenlebens organisieren, um die menschliche und planetarische Gesundheit zu schützen. Dies ist nur durch die Einbeziehung des Einzelnen und der Gemeinschaft und durch groß angelegte langfristige Makro-Interventionen erreichbar, die wirtschaftliche Investitionen in intelligente Städte, die Transformation der Energieerzeugung und die Abschaffung fossiler Brennstoffe umfassen. Der European Green Deal kann eine Chance sein, als umfassender Fahrplan der EU ressourceneffizienter und nachhaltiger zu sein, um Städte durch eine bessere Stadt- und Verkehrsplanung klimaneutral, lebenswerter und gesünder zu machen.

\section{Fazit}

In Städten konzentrieren sich Menschen und Ressourcen an einem Ort, was sowohl Gefahren als auch sehr reale Möglichkeiten zur Verbesserung der Gesundheit schafft. In dicht besiedelten und stark urbanisierten Gebieten sind Umwelteinflüsse prominente Merkmale des täglichen Lebens, welche die menschliche und planetarische Gesundheit systemisch beeinträchtigen können. Die moderne Städteplanung ist der Schlüssel, um die Gesundheitsprobleme der ungesunden urbanen Umgebung zu lösen.

Interessenkonflikt

Die Autorinnen/Autoren geben an, dass kein Interessenkonflikt besteht.
[1] Nieuwenhuijsen MJ. Urban and transport planning, environmental exposures and health-new concepts, methods and tools to improve health in cities. Environ Health 2016; 15 (Suppl 01): 38. doi:10.1186/s12940-0160108-1

[2] Munzel T, Sorensen M, Lelieveld J et al. Heart healthy cities: genetics loads the gun but the environment pulls the trigger. Eur Heart J 2021; 42: 2422-2438. doi:10.1093/eurheartj/ehab235

[3] Giles-Corti B, Vernez-Moudon A, Reis R et al. City planning and population health: a global challenge. Lancet 2016; 388: 2912-2924. doi:10.1 016/S0140-6736(16)30066-6

[4] Munzel T, Gori T, Al-Kindi S et al. Effects of gaseous and solid constituents of air pollution on endothelial function. Eur Heart J 2018; 39: 35433550. doi:10.1093/eurheartj/ehy481

[5] Lelieveld J, Pozzer A, Poschl U et al. Loss of life expectancy from air pollution compared to other risk factors: a worldwide perspective. Cardiovasc Res 2020; 116: 1910-1917. doi:10.1093/cvr/cvaa025

[6] Nieuwenhuijsen MJ. Influence of urban and transport planning and the city environment on cardiovascular disease. Nat Rev Cardiol 2018; 15: 432-438. doi:10.1038/s41569-018-0003-2

[7] Munzel T, Miller MR, Sorensen M et al. Reduction of environmental pollutants for prevention of cardiovascular disease: it's time to act. Eur Heart J 2020; 41: 3989-3997. doi:10.1093/eurheartj/ehaa745

[8] Munzel T, Schmidt FP, Steven S et al. Environmental Noise and the Cardiovascular System. J Am Coll Cardiol 2018; 71: 688-697. doi:10.1016/j.j acc.2017.12.015

[9] Falchi F, Cinzano P, Duriscoe D et al. The new world atlas of artificial night sky brightness. Sci Adv 2016; 2: e1600377. doi:10.1126/sciadv.1600377

[10] Sun S, Cao W, Ge Y et al. Outdoor Light at Night and Risk of Coronary Heart Disease among Older Adults: A Prospective Cohort Study. Eur Heart J 2021; 42: 822-830. doi:10.1093/eurheartj/ehaa846

[11] Nieuwenhuijsen MJ. Urban and transport planning pathways to carbon neutral, liveable and healthy cities; A review of the current evidence. Environ Int 2020; 140: 105661. doi:10.1016/j.envint.2020.105661

[12] Moreno C. The 15 minutes-city: for a new chrono-urbanism! Im Internet (Stand: 19.09.2021): http://www.moreno-web.net/the-15-minutes-cityfor-a-new-chrono-urbanism-pr-carlos-moreno/ 